\title{
The Motor KIF5C Links the Requirements of Stable Microtubules and IGF-1 Receptor Membrane Insertion for Neuronal Polarization
}

\author{
Mariana Oksdath $^{1}$ • Alvaro F. Nieto Guil ${ }^{1}$ - Diego Grassi ${ }^{1}$ - Lucas J. Sosa ${ }^{1}$ • \\ Santiago Quiroga ${ }^{1}$ (D)
}

Received: 30 March 2016 / Accepted: 19 September 2016

(C) Springer Science+Business Media New York 2016

\begin{abstract}
Three early signals of asymmetry have been described to occur in a single neurite of neurons at stage 2 of differentiation (before polarization) and shown to be essential for neuronal polarization: (i) accumulation of stable microtubules, (ii) enrichment of the plasma membrane with activatable IGF-1r, and (iii) polarized transport of the microtubular motor KIF5C. Here, we studied the possible relationship between these three phenomena. Our results show that the activatable (membrane-inserted) IGF-1r and stable microtubules accumulate in the same neurite of cells at stage 2 . The polarized insertion of IGF-1r depends on microtubule dynamics as shown using drugs which modify microtubule stability. Silencing of KIF5C expression prevents the polarized insertion of IGF-1r into the neuronal plasmalemma and neuronal polarization. Syntaxin 6 and VAMP4, necessary for the polarized insertion of the IGF-1r, are associated to vesicles carried by the microtubular motor KIF5C and is transported preferentially to the neurite where KIF5C accumulates. We conclude that the enrichment of stable microtubules in the future axon enhances KIF5C-mediated vesicular transport of syntaxin 6 and VAMP4, which in turn mediates the polarized insertion of IGF-1r in the plasmalemma, a key step for neuronal polarization. We herewith establish a mechanistic link between
\end{abstract}

Alvaro F. Nieto Guil and Diego Grassi contributed equally in this article.

Electronic supplementary material The online version of this article (doi:10.1007/s12035-016-0144-4) contains supplementary material, which is available to authorized users.

Santiago Quiroga

squiroga@fcq.unc.edu.ar

1 Departamento de Química Biológica-CIQUIBIC, Facultad de Ciencias Químicas, Universidad Nacional de Córdoba-CONICET, Haya de la Torre esquina Medina Allende, Ciudad Universitaria, 5000 Córdoba, Argentina three early polarity events necessary for the establishment of neuronal polarity.

Keywords Neuronal differentiation - Neuronal polarization · IGF-1 receptor · KIF5C · Stable microtubules · Syntaxin 6

\section{Introduction}

The formation of a polarized neuron, containing one long axon and several branching dendrites, requires the action of two interrelated processes, specification of the axon and axonal elongation. It has been shown that axon specification is defined early, at the time of generation of the first two, oppositely positioned, neurites [1]. After specification, initial axonal outgrowth is essential for neuronal polarization and is a particularly early event, occurring in neurons that have not yet exhibited a discernible axon (stage 2 of differentiation). Initial axonal outgrowth is regulated via the segregation of activatable (membrane-inserted) IGF-1 receptors (IGF-1r) in a single neurite $[2,3]$. Subsequently, upon activation of IGF1r, active phosphatidylinositol-3 kinase (PI3k) and its product, phosphatidylinositol 3,4,5-trisphosphate (PIP3), accumulate at the growth cone of the neurite. These events are critical for the outgrowth of the future axon $[4,5]$. In order to become activatable, the IGF-1r needs to be inserted to the neuron plasmalemma and expose the IGF-1 binding motifs to the extracellular space. This occurs by incorporation of exocytic vesicles containing IGF-1r with the participation of the exocyst complex [6] and the SNAREs proteins VAMP4, Syntaxin 6, and SNAP23 [7]. However, the molecular events responsible for the polarized insertion of IGF-1r to one neurite (out of four or five in hippocampal neurons) have not yet been described. Besides the polarized insertion of IGF-1r, two more early asymmetric events have been described in developing 
neurons: (i) the accumulation of stable microtubules in one single neurite (the future axon [8]) and (ii) the preferential transport to the specified neurite of the microtubular motor KIF5C [9] which shows a preference to interact with axonal over dendritic microtubules [10]. The experiments shown here were designed to determine the following: (i) if the three early events of polarization described during neural differentiation are independent or interdependent phenomena; and (ii) the molecular mechanism governing the polarized exocytic insertion of IGF-1r to a single neurite in neurons at stage 2 of differentiation. Our results show that the activatable IGF-1r and stable microtubules accumulate within the same neurite in cells at stage 2 of development. We also show that the accumulation of stable microtubules in the specified neurite is essential for the normal polarized insertion of IGF-1r, as demonstrated by using drugs which modify microtubules stability. Silencing expression of KIF5C prevents the polarized insertion of IGF-1r and, therefore, neuronal polarization. Moreover, the SNAREs Syntaxin 6 (Stx6) and VAMP4, necessary for the polarized insertion of IGF-1r and the establishment of neuronal polarity, are associated with vesicles carried by the microtubular motor KIF5C and transported preferentially to the neurite where $\mathrm{KIF} 5 \mathrm{C}$ is accumulated (the future axon). We conclude that the transport of Stx6 and VAMP4 by KIF5C to the specified neurite enables IGF-1r exocytosis and membrane insertion in that neurite. Moreover, because KIF5C exhibits preference for stable microtubules, this finding establishes the link between the requirement for stable microtubules, KIF5C enrichment and IGF-1r exocytosis for the establishment of neuronal polarization.

\section{Results}

1. Accumulation of stable microtubules and IGF-1r signaling are necessary for neuronal polarization

We first investigated if the neurite accumulating stable microtubules is the same as that exhibiting a significantly higher immunostaining to the activatable, membrane-inserted, IGF1r. For that purpose, we selected neurons at stage 2 of differentiation with five neurites and measured the fluorescence intensity (F.I.) of immunostaining with an antibody against acetylated tubulin as a marker of stable microtubules. The neurite with the highest F.I. ( $p \leq 0.0001)$ was assigned the number 1 , and the rest of the neurites were numbered 2 to 5 following a clockwise direction. We then measured the F.I. of immunostaining with an antibody against phosphorylated IGF-1r (pIGF-1r) of the five neurites. As shown in Fig. 1a, results indicated that the neurite number 1 exhibited also a significantly higher F.I. $(p \leq 0.00004)$ for pIGF-1r. We next investigated a possible relationship between the accumulation of stable microtubules and the polarized membrane insertion of IGF-1r by applying low doses [8] of the microtubuledestabilizing drug nocodazole or the microtubule-stabilizing drug taxol and studying the distribution of pIGF-1r in these cells. As expected, in control untreated cells stimulated with IGF-1, we observed a polarized distribution of the activated IGF-1r to a single neurite (Fig. 1b, arrow). In contrast, neurons treated with nocodazole exhibited lower intensity labeling of the pIGF-1r not confined to any particular neurite (Fig. 1b). In turn, treatment with taxol produced a more intense labeling of pIGF-1r in all the neurites (Fig. 1b). To quantify these differences, we calculated an "active IGF-1r polarization index" (see legend to Fig. 1b). As shown in Fig. 1c, this index was significantly higher $(p \leq 0.004)$ in the control neurons than in the neurons treated with nocodazole or taxol. In contrast, treatment with the actin-depolymerizing drug cytochalasin D did not affect the pIGF-1r distribution (Fig. 1b, c). Cells were subjected to the same experimental conditions and stained with $\beta$ gc antibody, which recognizes both the phosphorylated and non-phosphorylated forms of the IGF-1r [11]. Control and nocodazole- or taxol- treated neurons at stage 2 (12 h in culture) exhibited intracellular $\beta$ gc labeling in all neurites (Fig. S1) with no obvious effects of either nocodazole or taxol on the subcellular localization of IGF-1r.

To investigate a direct relationship between the polarized distribution of membrane-inserted IGF-1r microtubule dynamics and the establishment of neuronal polarity, we cultured hippocampal neurons under control conditions or treated with nocodazole or taxol as described and stimulated with $20 \mathrm{nM}$ IGF-1. The control medium contained DMSO and $5 \mathrm{nM}$ insulin, an amount sufficient to guarantee neuronal survival without stimulating IGF-1 receptors [12]. As expected, most neurons under control conditions exhibited a unique axon (Tau-1 positive) after $36 \mathrm{~h}$ of D.I.V. when stimulated with IGF-1 (Fig. 2a). In contrast, many cells treated with taxol or cytochalasin $\mathrm{D}$ exhibited more than one axon under the same culture conditions (Fig. 2a). Most cells remained arrested at stage 2 of differentiation in cultures treated with nocodazole (Fig. 2a). To quantify these observations, we scored the differentiation stages of neurons cultured either under control conditions or treated with taxol, nocodazole, or cytochalasin $\mathrm{D}$, respectively, after $36 \mathrm{~h}$ in vitro. We found that over $75 \%$ of the control, neurons stimulated with IGF-1 showed an identifiable, tau-1-containing axon (Fig. 2b). In contrast, around $25-30 \%$ of the cells treated with taxol or cytochalasin D and stimulated with IGF-1 exhibited multiple axons (note that cells treated with taxol or cytochalasin D in control conditions without IGF-1 did not form any axon, indicating that besides enrichment of stable microtubules or actin depolimerization, stimulation with IGF-1 is necessary to stimulate axonal outgrowth; see also Fig. 2a) and most cells treated with nocodazole and stimulated with IGF-1 remained at stages 1 or 2 of differentiation; less than $30 \%$ had formed a discernible axon (Fig. 2b). 

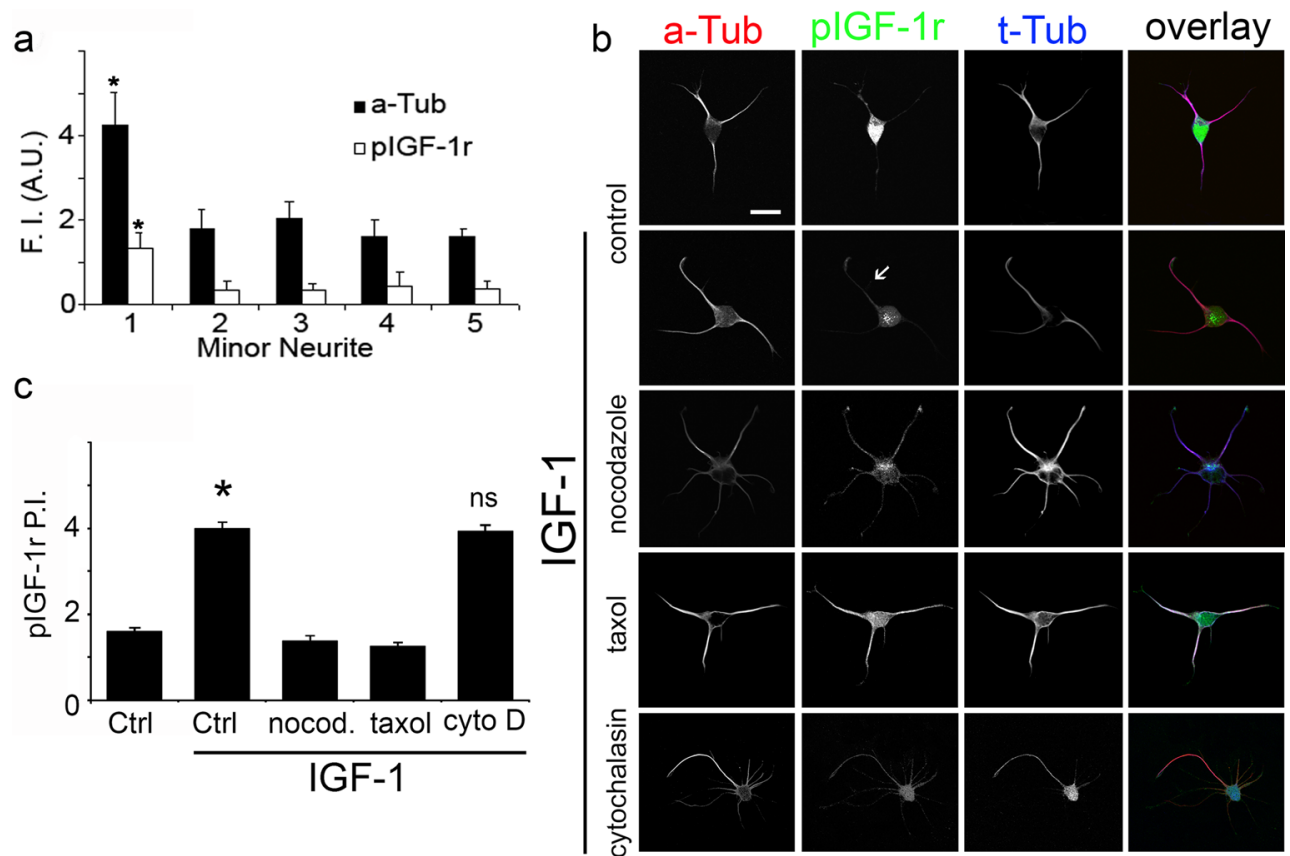

Fig. 1 Microtubule stability is important for pIGF-1r polarization to a single neurite in neurons at Stage 2 of differentiation. a Fluorescence intensity (F.I. \pm s.e.m.) measured in neurons at stage 2 of differentiation, stained with antibodies against acetylated tubulin (black bars) and pIGF$\operatorname{1r}$ (empty bars). Note that the neurite number 1 , showing accumulation of acetylated tubulin, exhibited also a significantly higher F.I. for pIGF-1r ( $p \leq 0.0001$ by one way ANOVA post-Hoc Tukey). $n=3$ independent experiments, at least 10 cells were analyzed in each experiment. b Triple immunofluorescence micrographs of rat hippocampal neurons cultured for $12 \mathrm{~h}$ with DMSO in control conditions ( $5 \mathrm{nM}$ insulin-top) or stimulated with $10 \mathrm{nM}$ IGF-1 (second from top to bottom) and treated with $45 \mathrm{nM}$ nocodazole (third from top), $3 \mathrm{nM}$ Taxol (fourth from top) or $0.5 \mu \mathrm{g} / \mathrm{ml}$ cytochalasin D (bottom) and stained with antibodies to pIGF1r (green), acetylated tubulin (red) and tyrosinated tubulin (blue). Note

the polarization of active (membrane-inserted) IGF-1r to one of the minor neurites of the cell cultured in the presence of DMSO and stimulated with IGF-1 (second from top) or stimulated with IGF-1 and treated with cytochalasin D (bottom). In contrast, neurons treated with nocodazole (third from top) or taxol (fourth from top) failed to polarize the active IGF-1r. c A "polarization index" of active IGF-1r (IGF-1r P.I.) was calculated as the fluorescence intensity (A.U.) of the brightest minor neurite/average fluorescence intensity (A.U.) of the other minor neurites of the same cell. The polarization index is significantly higher in the neurons cultured in the presence of DMSO and stimulated with IGF-1 (* $p \leq 0.004$ by test " $t$ ") compared to those cultured with nocodazole or taxol. No differences in the P.I. index were found between the control cells and the cells treated with cytochalasin D. $N=3$ independent experiments, at least 10 cells were analyzed for each condition. Calibration bar $=50 \mu \mathrm{m}$

2. The microtubular motor KIF5C is necessary for neuronal polarization and directed IGF-1r insertion

To study the possible involvement of the microtubular motor KIF5C in the regulation of initial axonal outgrowth and the establishment of neuronal polarity, we silenced the expression of KIF5C using a targeted shRNA inserted into a dicistronic plasmid also encoding enhanced green fluorescent protein (GFP). Transfection of cell cultures with KIF5C-targeted shRNA significantly and specifically decreased KIF5C protein (Fig. 3c). The transfected neurons expressed virtually no detectable KIF5C and failed to form axon-like processes; only short, minor neurites were present (Fig. 3a). In contrast, neurons transfected with a scrambled RNA (ssRNA) sequence inserted in the same plasmid exhibited normal levels of KIF5C and generated a long axon-like process (Fig. 3a). Cotransfection of neurons with KIF5C-targeted shRNA and an RFP-tagged wild-type form of KIF5C (mouse) rescued the phenotype and induced the outgrowth of an axon-like process

(Fig. 3a), indicating that the lack of polarization of the shRNA-treated neurons is not due to off-target effects. To quantify this observation, we scored the differentiation stages of neurons transfected with KIF5C-targeted shRNA compared to neurons transfected with ssRNA after $40 \mathrm{~h}$ in vitro. We found that over $70 \%$ of the transfected neurons remained at stages 1 or 2 of differentiation, and less than $30 \%$ had formed a discernible axon. In contrast, around $70 \%$ of the control ssRNA transfected neurons showed an axon-like long process (Fig. 3b). An early event of the establishment of neuronal polarity during neuronal differentiation is the enrichment of activatable IGF-1r in a single minor neurite at stage 2 of differentiation [2]. In order to be activated, the IGF-1r needs to be inserted into the neuronal plasmalemma so that the ligand binding site is exposed to the extracellular space. Therefore, we next studied the consequences of loss of function of $\mathrm{KIF} 5 \mathrm{C}$ on the enrichment of active IGF-1r in the plasmalemma of a single neurite in neurons at stage 2 of differentiation, as described above. We observed the expected polarized distribution of the activated IGF-1r (Fig. 4a top) in stage 2 

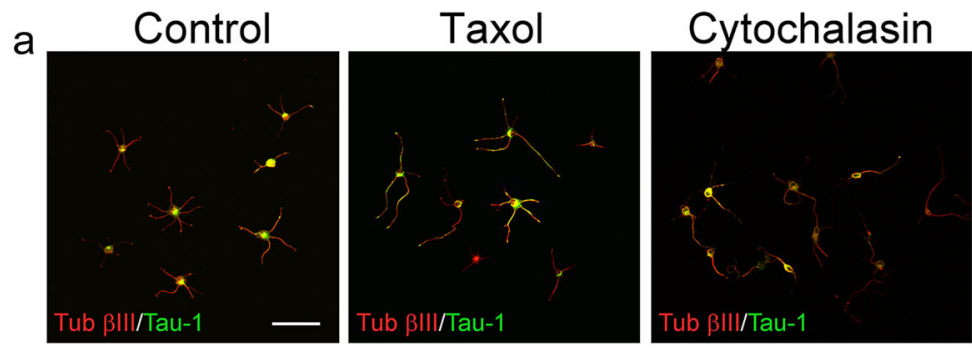

IGF-1
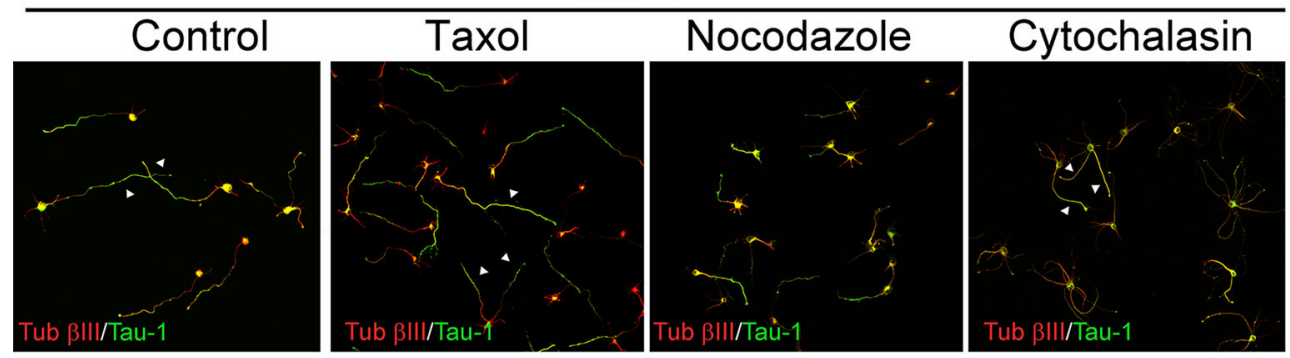

b
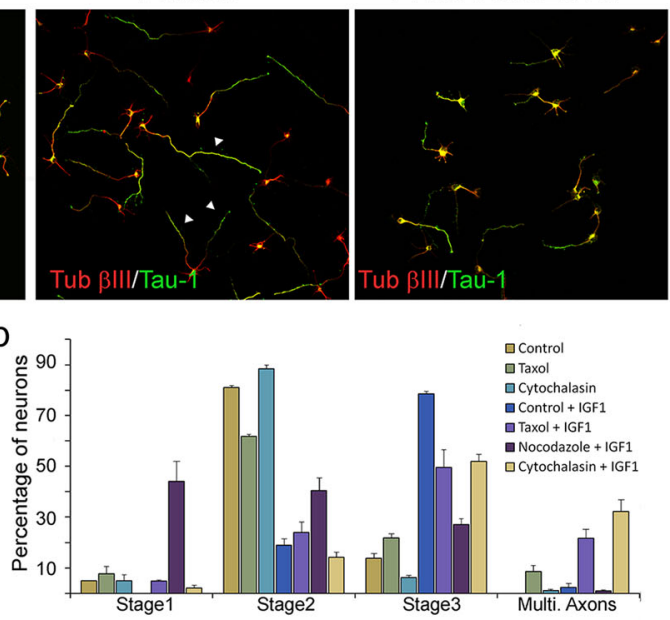
in culture. a Double immunofluorescence micrographs of hippocampal neurons after $36 \mathrm{~h}$ in culture showing the distribution of the neuronal marker $\beta$-III tubulin (red) and the axonal marker Tau-1 (green). Cells were cultured in medium with a low concentration of insulin $(5 \mathrm{nM}$ insulin-top lane) or stimulated with $20 \mathrm{nM} \mathrm{IGF-1} \mathrm{(bottom} \mathrm{lane)} \mathrm{and} \mathrm{treated}$ with Taxol (second from left), nocodazole (third from left), or cytochalasin D (right), respectively. Most cells challenged with IGF-1 exhibited long, Tau-1 positive axons. In contrast, cells treated with nocodazole remained

neurons transfected with a ssRNA sequence (14 h in vitro), deprived of growth factor for $5 \mathrm{~h}$, and stimulated for $5 \mathrm{~min}$ with $20 \mathrm{nM}$ IGF-1. In contrast, neurons transfected with KIF5C (Fig. 4a, bottom) targeted shRNA exhibited labeling of the activated IGF-1 $\mathrm{r}$ that was not confined to any particular minor process. The "active IGF-1r polarization index" (described in the legend of Fig. 1) was significantly higher $(p \leq 0.0005)$ in the neurons transfected with the ssRNA sequence than in the KIF5C-suppressed neurons (Fig. 4b).

3. KIF5C transports the SNARE protein Stx6 into the future axon

These results prompted us to investigate if the microtubular motor KIF5C could transport a protein(s) necessary for the exocytotic insertion of the IGF-1r to the plasma membrane of a selected neurite. Immunoprecipitation of microsomes from rat fetal brain using an anti-KIF5C antibody showed that two SNARE proteins, Stx6 and VAMP4, are present in the

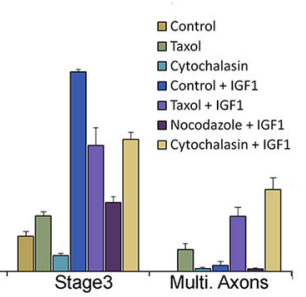

arrested at stage 2 of differentiation. Taxol or cytochalasin D treated cultures exhibited cells with multiple axons when challenged with IGF1. b Percentage (+s.e.m.) of neurons at different stages of differentiation grown for $36 \mathrm{~h}$ under the same conditions described in $\mathbf{a}$. Note that most cells cultured in the presence of IGF-1 are in stage 3 of differentiation (polarized). Around $30 \%$ of the cells cultured in high insulin plus taxol or cytochalasin D exhibited multiple axons. $n=3$ independent experiments. One hundred or more cells were scored in each condition. Calibration bar $=100 \mu \mathrm{m}$

vesicles also containing KIF5C (Fig. 5a). In contrast, other proteins such as IGF-1r and the proteins related to the exocyst complex TC10, Exo70, sec5, and sec8 plus the SNARE protein SNAP23 (note that these proteins are involved in the exocytotic insertion of IGF-1r to the plasmalemma) $[6,7]$ did not co-immunoprecipitate with KIF5C (Fig. 5a). For IGF-1r, this result was expected, since IGF-1r is carried by the microtubular motor KIF2 [13] with the participation of rab10 and Marcks [14]. To study the possible co-existence of Stx6 and VAMP4 in the same population of vesicles, we performed immunoprecipitation of fetal rat microsomes with antibodies to Stx6 or VAMP4. The results of these experiments showed that VAMP4 was present in vesicles immunoprecipitated with Stx6 antibody (Fig. 5b, top) and that vesicles containing Stx6 were also immunoprecipitated using an anti-VAMP4 antibody (Fig. 5b, bottom). To obtain direct evidence about Stx6 and VAMP4 transport by KIF5C in neurons, we cultivated pyramidal hippocampal neurons for 14 $16 \mathrm{~h}$ (cells were already polarized) and, at this time, transfected the cultures with a shRNA directed to KIF5C 
Fig. 3 KIF5C is essential for hippocampal neurons polarization. a Immunofluorescence micrographs of hippocampal neurons after $24 \mathrm{~h}$ in culture showing the localizations of KIF5C (red) GFP was a transfection marker (first two rows). Note that the neurons transfected with KIF5C-targeted shRNA (second row) did not develop axons. Co-transfection with KIF5C-targeted shRNA plus wild-type, RFP-tagged KIF5C (red) rescued the phenotype. $\mathbf{b}$ Relative percentages $( \pm$ s.e.m.) of control neurons or neurons containing KIF5C-targeted shRNA at specific stages of differentiation after $40 \mathrm{~h}$ in culture. $n=3$ independent experiments. At least 100 neurons were scored for each condition ( $p \leq 0.001$ by Test " $t$ "). $\mathbf{c}$ Western blots showing KIF5C levels in rat brain glioma cells C6 (ATCC CCL 107 were tested for contamination following institutional protocols) untransfected (left) transfected with ssRNA (middle), and a KIF5C-targeted shRNA (right). Tubulin was used as a loading control. Calibration bar $=50 \mu \mathrm{m}$ a
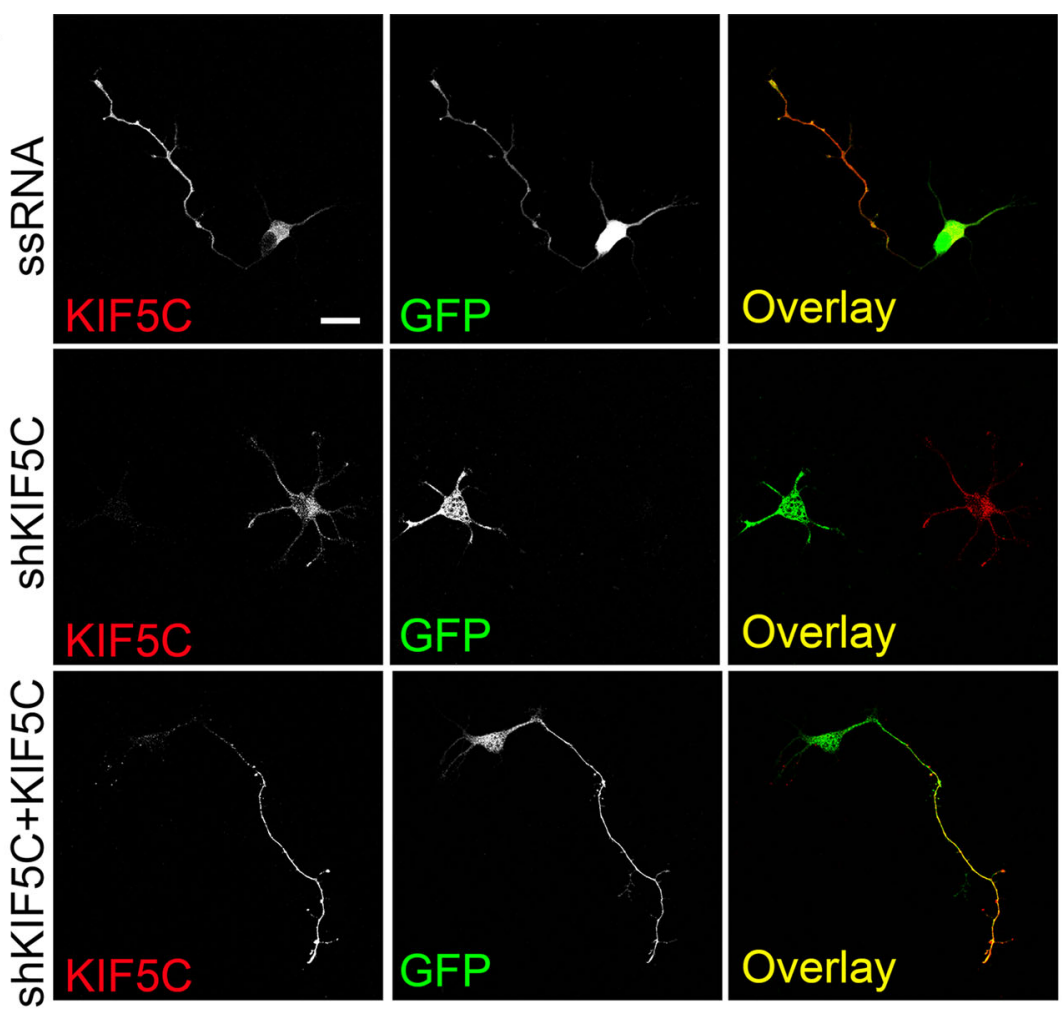

b

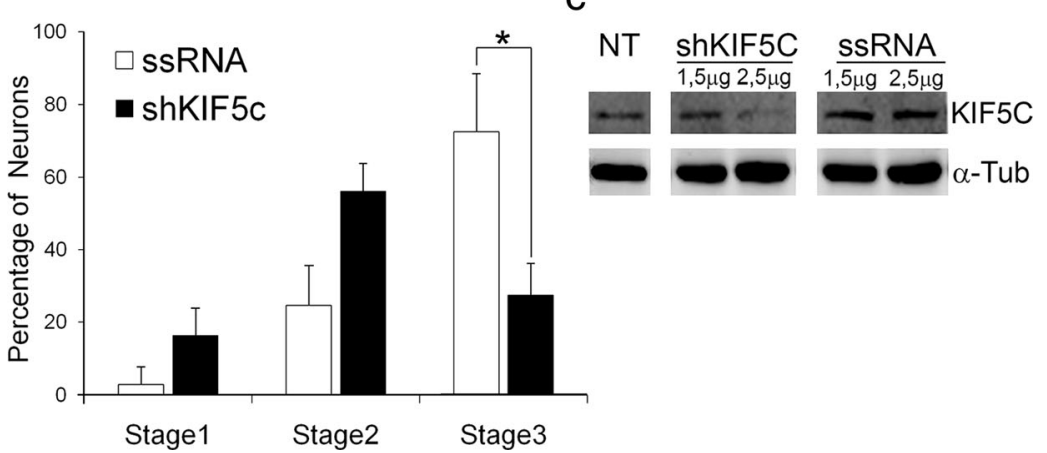

(efficiency of the shRNA is shown in Fig. 3c) or a control ssRNA and cultured the cells for further $24 \mathrm{~h}$. At this time, the neurons were fixed and double immunostained with antibodies to Stx6 or VAMP4 plus Tau-1. Silencing of KIF5C produced a noticeable decrease of Stx6 staining in axons (Fig. 6a), which exhibited a relatively prominent expression in neurons transfected with the ssRNA (Fig. 6a). In contrast, no evident changes in the distribution of VAMP4 were observed in cells silenced for KIF5C compared to controls (Fig. 6a). In view of these results and to further study the association of Stx6 and VAMP4 with KIF5C, microsomes were fractionated by isopycnic centrifugation in linear sucrose density gradients and analyzed by Western blots with antibodies to KIF5C, Stx6, and VAMP4 (SNAP23 was also included as a non-co-immunoprecipitating protein). Results are shown in Fig. $6 \mathrm{~b}$ and indicated a strong co-distribution of KIF5C, Stx6, and VAMP4 along the gradient. However, the distribution of VAMP4 was wider, with enriched fractions which did not co-migrate with KIF5C and Stx6. As expected, there is little co-localization between KIF5C and SNAP23 (Fig. 6b). It follows that KIF5C seems to transport vesicles containing Stx6 and VAMP4, and that the latter SNARE could be also driven in other vesicle population by a different (yet unidentified) motor.

These last results indicate that KIF5C can be responsible for the transport of Stx6 and VAMP4 (necessary for the exocytotic insertion of the IGF-1r) [7] and trigger the insertion to the membrane of the IGF-1r in one neurite in neurons in stage 2. To fulfill this role, KIF5C should be able to transport Stx6 (and VAMP4 present in the same population of vesicles) preferentially to one neurite (the future axon) in cells not yet polarized. To study this possibility, we used dual color spinning disk microscopy of neurons in stage 2 transfected with GFP-tagged Stx6 plus KIF5C ${ }^{560}$ TdTomatoe as a marker of the 


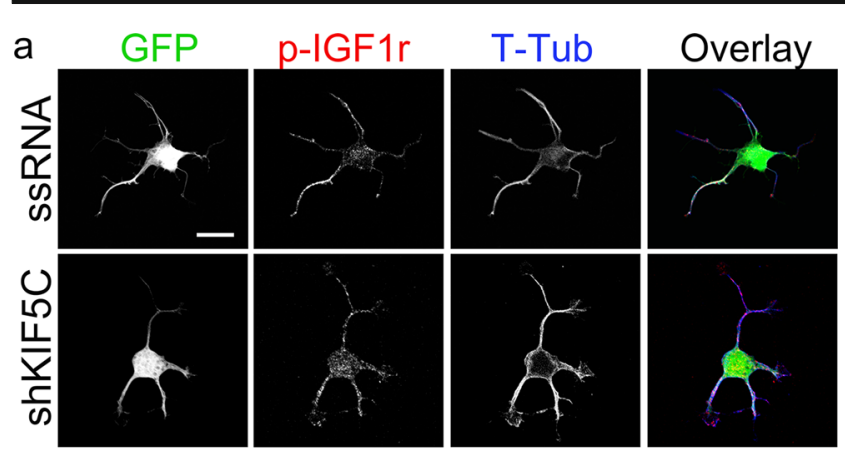

b

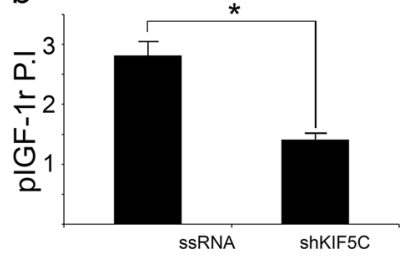

Fig. 4 KIF5C is necessary for the polarization of activatable IGF-1r to a single neurite in cells at stage 2 of differentiation. a Double immunofluorescence micrographs of hippocampal neurons (after $14 \mathrm{~h}$ in culture) showing the distributions of tyrosinated tubulin (blue), phosphorylated IGF-1r (red), and the transfection marker GFP. Neurons were transfected with either a scrambled RNA sequence (ssRNA-top) or KIF5C-targeted shRNA (second row). Neurons were deprived of growth factors for $4 \mathrm{~h}$ and stimulated with $20 \mathrm{nM}$ IGF-1 for $2 \mathrm{~min}$ before fixation. Note the polarization of active (membrane-inserted) IGF-1r to one of the minor neurites of the cell transfected with ssRNA (arrow-top). In contrast, neurons transfected with $\mathrm{KIF} 5 \mathrm{C}$ (second rows) failed to polarize the active IGF-1r to any neurite. b A "polarization index" of active IGF-1r (IGF-1r P.I.) was calculated as the fluorescence intensity (A.U.) of the brightest minor neurite/average fluorescence intensity (A.U.) of the other minor neurites of the same cell. Neurons were processed as in a. The polarization index is significantly higher in neurons transfected with scrambled sequence $\operatorname{shRNA}(* p \leq 0.0005$ by Test " $t$ ") compared to those transfected with KIF5C shRNA. $n=3$ independent experiments 10 cells were scored for each condition. Calibration bar $=20 \mu \mathrm{m}$

future axon [9]. By measuring anterograde transport of vesicles containing Stx6 in the minor neurites from the cell body to the tip (see "Materials and Methods" section), we determined that its rate of transport to the future axon (neurite 1) is significantly higher ( $p \leq 0.0001$ by ANOVA) compared to the transport to the remaining neurites (2 and 3, Fig. 7a, c) As a control, we also measured anterograde transport of the dendritic protein transferrin receptor, which we expected to be transported preferentially to the remaining neurites $(2,3$, and 4) and not to neurite 1 enriched in KIF5C [15]. In fact, Fig. 7b, c shows the "dendritic preference" of the transferrin receptor.

\section{Discussion}

The establishment of neuronal polarity is driven by extracellular and intracellular events related to multiple cellular functions. Regarding extracellular clues, it has been shown that the activation of IGF-1r by its ligand IGF-1 is important to regulate this process in hippocampal pyramidal neurons in culture
[2] and motor neurons in vivo [16]. The intracellular mechanisms include changes in the stability of microtubules impacting their function $[8,17]$. Axon formation requires directed transport of membrane vesicles and other cargos along polarized microtubules [17-20]. Polarized transport by anterograde-directed motors, such as KIF5 and KIF3 plays a central role in establishing a single axon $[9,21]$.

In this context, three early signals of asymmetry have been described in a single neurite of neurons at stage 2 of differentiation, preceding morphological polarization. These signals were shown to be essential for the establishment of neuronal polarity: (i) the accumulation of stable microtubules [8]; (ii) the enrichment of activatable, membrane-inserted, IGF-1r [2]; and (iii) the preferential transport of the microtubular motor KIF5C [9]. Herein, we aimed at establishing a mechanistic link between these three phenomena. Our results indicate that the accumulation of stable microtubules in a specified neurite is necessary for the enrichment of activatable IGF-1r in the same neurite and axonal outgrowth. Indeed, the microtubuledestabilizing agent nocodazole and taxol, a drug that causes accumulation of stable microtubules in all the neurites [8] inhibited both the polarized distribution of activatable IGF1r and axonal outgrowth, necessary for the establishment of neuronal polarity (Figs. 1 and 2). Interestingly, cytochalasin $\mathrm{D}$, an actin-depolarizing drug able to produce neurons with multiple axons [22], did not affect the distribution of activatable IGF-1r (Figs. 1 and 2). It follows that the enrichment of IGF-1r in one neurite depends on microtubule dynamics but not on actin filaments stability. Knockdown of the microtubular motor KIF5C, required for the establishment of neuronal polarity (Fig. 3), also impeded the polarized distribution of activatable IGF-1r (Fig. 4). This is in close relation to the fact that Stx6 and VAMP4, two SNAREs required for the polarized insertion of IGF-1r and the establishment of neuronal polarization (see [7]), are associated with vesicles transported by KIF5C (Figs. 5 and 6). Finally, we demonstrated by using two color spinning disk microscopy, that Stx6 is transported preferentially to the process where KIF5C is accumulated (Fig. 7).

We propose that KIF5C drives Stx6 and VAMP4 preferentially to one process, "walking" on stable microtubules (see [10]). Once Stx6 and VAMP4 accumulate at the neurite enriched in stable microtubules, it is able to trigger the insertion, by exocytosis, of IGF-1r in the neuritic plasmalemma. Upon insertion in the membrane, IGF-1r can be activated by IGF-1 and may induce membrane insertion of more IGF-1r [6]. All together, these molecular events generate a selfreinforcing mechanism deemed necessary for initial axonal outgrowth and the establishment of neuronal polarity. We think that our study provides a comprehensive understanding of the participation of the IGF-1r, microtubule dynamics, and KIF5C activity in the establishment of neuronal polarity. A cartoon illustrating the interconnection between these 


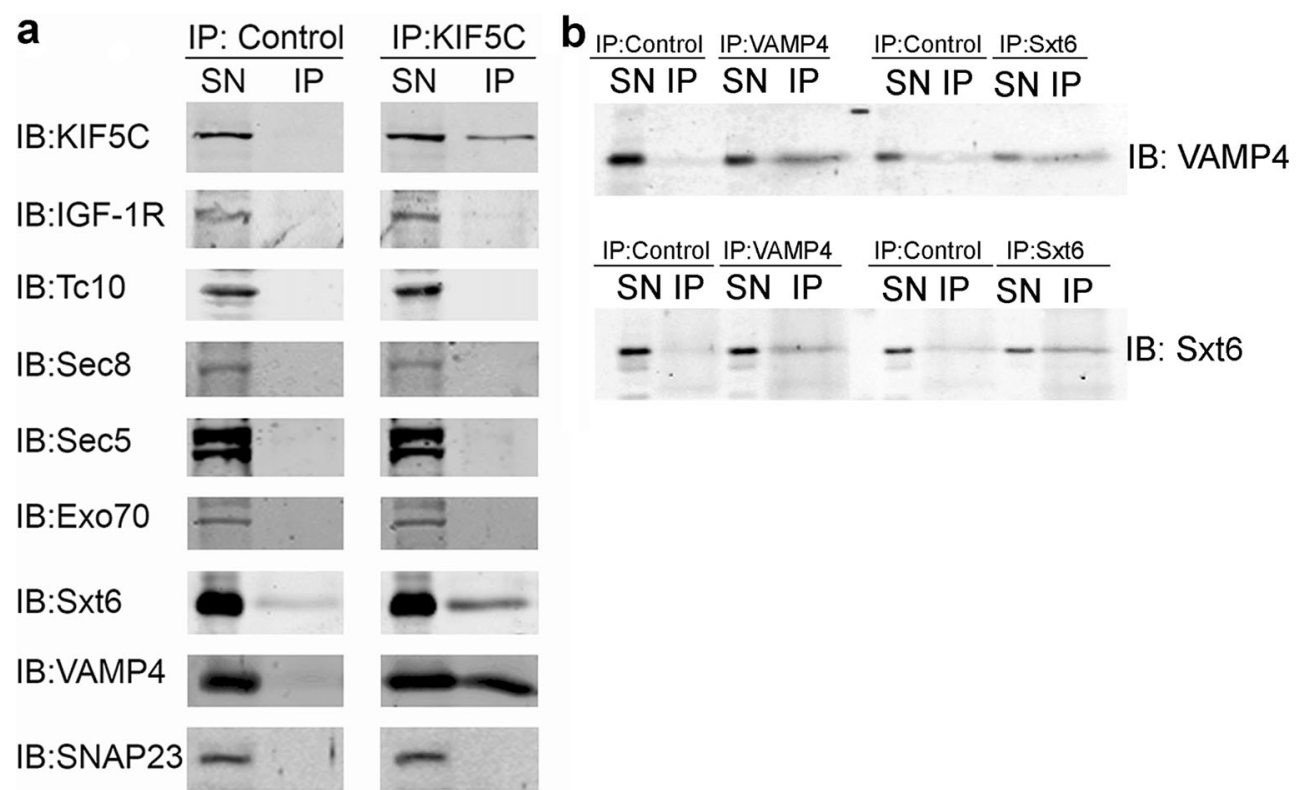

Fig. 5 KIF5C-associated vesicles contain Stx6 and VAMP4 as cargo proteins. a Western blots of microsomes immunoprecipitated in the absence of any relevant antibody (left) or with KIF5C antibody (right). The blots were probed with the following antibodies: anti-KIF5C, antiIGF-1r, anti-TC10, anti-Sec8, anti-Sec5, anti-Exo70, anti-Syntaxin 6,

different actors of neuronal polarization is shown in Fig. 8. More investigation is needed to determine which motor protein(s) are involved in the transport of TC10 and Exo70, two proteins that are also involved in the exocytosis of vesicles containing the IGF-1 receptor [6]. Relevant published information in relation with the early regulation of neuronal polarization also include the following: (i) inhibition of the chaperone protein Hsp90 within the first hours of culture affects polarity in hippocampal neurons and induces changes in KIF5C subcellular localization [24]) and (ii) c-Jun N-terminal kinase (JNK) activation is necessary for axogenesis and the establishment of neuronal polarity [23]. The precise coordination of JNK and Hsp90 activities plus the IGF-1r membrane insertion and activation inside an early time-window could be essential for neuronal polarization [24].

\section{Materials and Methods}

\section{Short Hairpin RNA Plasmids}

The shRNA sequences used as targets were as follows: KIF5C, 5'-cagcagaagaatggaagaa-3'. For control, a sequence of scrambled shRNA was used: $5^{\prime}$-agacaatgaagcgaagaag-3'. The resulting plasmids were referred to as shKIF5C and ssRNA (scrambled shRNA), respectively. All oligonucleotides were synthesized by SIGMA (MO, USA) and subcloned into pSuperNeo+GFP vector (Oligoengine).
anti-VAMP4, and anti-SNAP23, respectively (from top to bottom). b Western blots of microsomes immunoprecipitated in the absence of relevant antibodies (control), with an antibody to VAMP4 (left) or with an antibody to Stx6 (right). And probed with antibodies to VAMP4 (top) or Stx6 (bottom). SN supernatant, IP immunoprecipitate

\section{Primary Antibodies}

The following primary antibodies were used: rabbit polyclonal antibody to KIF5C (Cat. ab5630, ABCAM [25]), diluted to $0.8 \mu \mathrm{g} / \mu \mathrm{l}$ for immunofluorescence (IF), 1:4000 for immunoprecipitation (IP) and 1:2000 for Western blot (WB); rabbit polyclonal antibody to VAMP4 (Synaptic System, Cat 136,002 [26]), diluted to 1:100 (IF), 1:250 (WB); 1:200 (IP); mouse polyclonal antibody to Exo70 (Santa Cruz, ZZ7, Cat. sc-100733 [27]), 1:250 (WB); mouse monoclonal antibody to Sec8 (Santa Cruz, LB-2 Cat. sc-100734 [28]), 1:400 (WB); rabbit polyclonal antibody to Syntaxin 6 (Synaptic System, Cat. 110,062 [29]), diluted to 1:150 (IF), 1:600 (WB), and 1:250 (IP); rabbit polyclonal antibody to SNAP23 (Synaptic System, Cat 111,202 [30]), diluted to 1:50 (IF) and 1:200 (WB); goat polyclonal antibody to TC10 (Santa Cruz, E13, Cat. sc-26592 [31]), diluted to 1:150 (WB); rabbit polyclonal antibody $\beta \mathrm{gc}$ to IGF-1r $[2,11]$, diluted to $1: 100$ (IF) and 1:200 (WB); rabbit polyclonal antibody to phosphorylated (Tyr 980) IGF-1r (Cell Signaling, Cat. C14A11 [2]), diluted to 1:50 (IF); rabbit polyclonal antibody to $\beta$ III-tubulin (SigmaAldrich, Cat: T2200 [32]), diluted to 1:4000 (IF); mouse monoclonal antibody to the axonal marker Tau-1 (clone PC1C6, Cat. MAB3420 Millipore, [33]), diluted to 1:600 (IF); rat monoclonal antibody to tyrosinated tubulin (clone Tub-IA2, Sigma-Aldrich Cat. T9028 [34]), diluted to 1:2000 (IF); mouse monoclonal antibody to $\alpha$-tubulin (clone DM A1, Thermo Scientific, Cat. 62204), diluted to 1:2000 (WB); and mouse monoclonal to acetylated tubulin (clone 6-11B-1, Sigma, Cat T6793 [35]), diluted to 1:4000 (IF). 

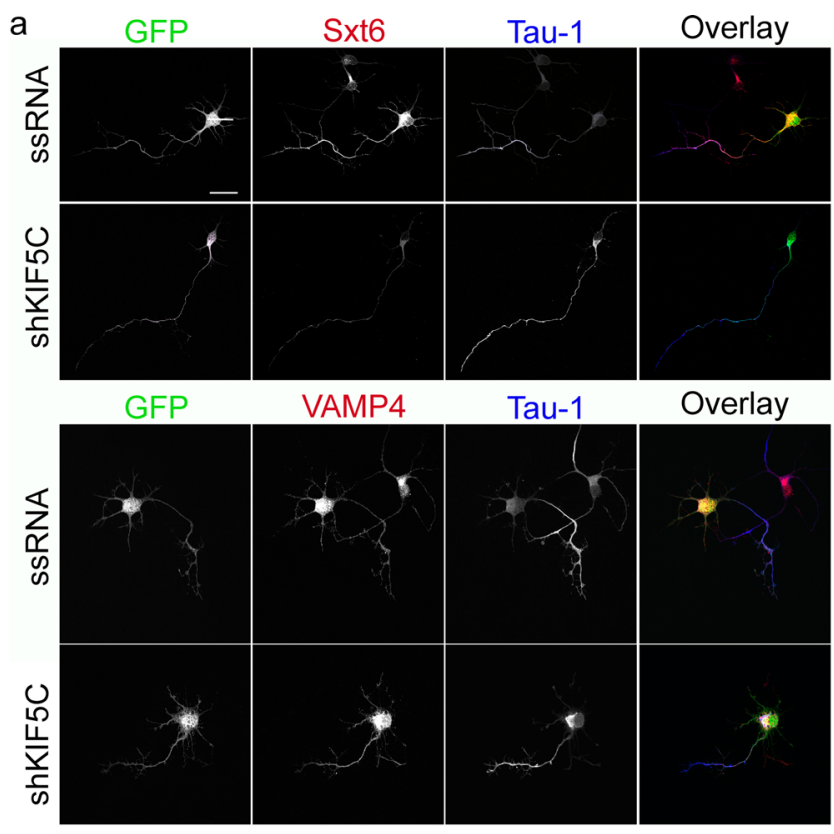

b

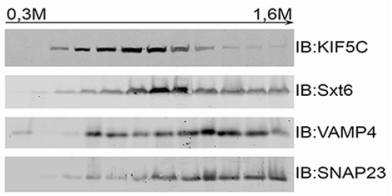

Fig. 6 KIF5C is essential for the transport of Stx 6 to axons. a Hippocampal neurons (after $12 \mathrm{~h}$ in culture) transfected with KIF5Ctargeted shRNA or ssRNA and cultured for $24 \mathrm{~h}$ after transfection. Immunofluorescence micrographs show the localizations of Stx6 or VAMP4 (red), Tau-1 (blue), and GFP as a transfection marker. Note that the neurons transfected with KIF5C-targeted shRNA (second lane) exhibited a noticeable decrease of Stx6 staining in axons. In contrast, no changes in the localization of VAMP4 were observed in cells silenced for KIF5C. Calibration bar $=50 \mu \mathrm{m}$. b Microsomes were separated across a continuous sucrose gradient extending from 0.3 to $1.6 \mathrm{M}$ sucrose by isopycnic centrifugation $(242000 \times g$ for $2 \mathrm{~h})$. Note the precise colocalization of KIF5C with Stx6. VAMP4 also co-localized with KIF5C and Stx6 (as expected) but showed a wider distribution in the gradient

\section{Culture and Transfection}

Dissociated hippocampal pyramidal neurons were prepared from fetal rat brain and cultured as described previously [2]. Briefly, cells were plated into polylysine-coated glass coverslips and maintained in DMEM plus $10 \%$ horse serum for $1 \mathrm{~h}$. The coverslips with the attached cells were subsequently transferred to 35 $\mathrm{mm}$ Petri dishes containing serum-free neurobasal medium plus the N2 mixture. Cultures were maintained in a humidified $37^{\circ} \mathrm{C}$ incubator with $5 \% \mathrm{CO}$. Shortly after plating, hippocampal neurons first extend lamellipodia (stage 1) and afterward several minor neurites that are initially indistinguishable (stage 2). Then, at stage 3 , one of these initially equivalent neurites grows more rapidly than the others and becomes the axon, whereas the other neurites subsequently develop into dendrites (stage 4). Neurons are considered to be at stage 3 when the length of the axon exceeds that of the average minor neurite by at least $20 \mu \mathrm{m}$. Transient transfection of cultured neurons was performed as described previously [7], and the constructs used at a concentration of $2 \mu \mathrm{g} / \mu \mathrm{l}$. For those experiments involving expression of shRNA sequences in early stages of neuronal development, a protocol of transfection of neurons in suspension, before plating, was used. It was similar to in-tube procedure described, with modifications. Briefly, DNA-Lipofectamine 2000 complex diluted in OPTIMEM $(80 \mu \mathrm{l})$ was made in a $1.5-\mathrm{ml}$ eppendorf tube. Five hundred nanograms of DNA and $1 \mu$ l of Lipofectamine 2000 were mixed in each reaction. This mixture was incubated at room temperature for $30 \mathrm{~min}$. Thereafter, $15 \mu \mathrm{l}$ of a neuron suspension $\left(6 \times 10^{4}\right.$ cells $)$ diluted in OPTIMEM was added. The cells-DNALipofectamine 2000 mixture was immediately plated over polylysine-coated glass coverslips and cultures were placed to $37{ }^{\circ} \mathrm{C}$ in a humidified $5 \% \mathrm{CO} 2$ incubator. After $1 \mathrm{~h}$, the transfecting complex/OPTIMEM was removed carefully from each coverslip (at this time most neurons were already attached) and DMEM containing $10 \%$ horse serum was added to cultures for 1 h at $37^{\circ} \mathrm{C}$. Finally, DMEM containing $10 \%$ horse serum was replaced for serum-free medium plus the $\mathrm{N} 2$ mixture. Cultures were maintained in a humidified $37^{\circ} \mathrm{C}$ incubator with $5 \% \mathrm{CO} 2$ for $24 \mathrm{~h}$ before fixation.

\section{Treatments with Taxol, Nocodazole or Cytochalasin D}

Depending on the experiment, $3 \mathrm{nM}$ taxol (Sigma-Aldrich) or $45 \mathrm{nM}$ or $3 \mu \mathrm{M}$ nocodazole (Sigma-Aldrich) were added to cultured neurons 6 or $24 \mathrm{~h}$ after plating and cells were further incubated for 12 or $24 \mathrm{~h}$ at $37^{\circ} \mathrm{C}$. For some experiments, cells were treated with nocodazole or $0.75 \%$ DMSO for $30 \mathrm{~min}$ and then simultaneously extracted and fixed to remove unpolymerized tubulin subunits and allow clear visualization of MTs. $0.5 \mu \mathrm{g} / \mathrm{ml}$ cytochalasin D (Sigma-Aldrich) was added to the cultured neurons just after plating and cells were further incubated for $36 \mathrm{~h}$ at $37^{\circ}$.

\section{Immunofluorescence Microscopy}

Cells were fixed for $20 \mathrm{~min}$ at room temperature with $4 \%(w /$ v) paraformaldehyde in PBS containing $4 \%(w / v)$ sucrose. Cultures were washed with PBS, permeabilized with $0.1 \%$ $(v / v)$ Triton X-100 in PBS for 6 min, washed in PBS again, and blocked for $1 \mathrm{~h}$ at room temperature. After labeling with a first primary antibody (overnight at $4{ }^{\circ} \mathrm{C}$ ) and washing with PBS, cultures were incubated with fluorescent secondary antibody conjugated to Alexa Fluor 488, 546, or 633 (1 h at room temperature) and washed with PBS. The cells were visualized using a spectral confocal microscope (Olympus FV1000). Images were captured and digitized using Olympus Fluoview Viewer software. For some experiments (see Fig. 6), cells were observed with a spinning disk microscope Yokogawa CSU-X1. Images were captured using an EMCCD camera Photometrics QuantEM 512 and a resolutive CCD camera Photometrics HQ2. Images were digitized using 


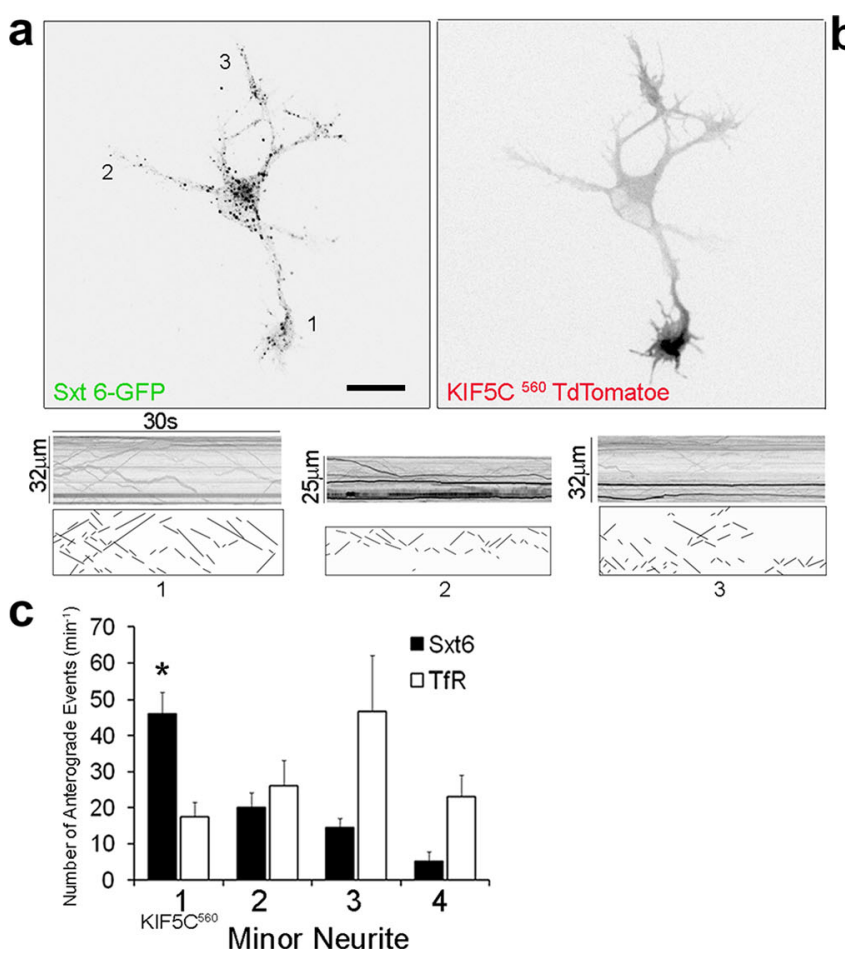

Fig. 7 Stx6 is transported preferentially to the future axon in cells at stage 2 of differentiation. a Neurons at stage 2 of differentiation were coelectroporated with Stx6-GFP (left) and $\mathrm{KIF}^{5} \mathrm{C}^{560}$ tdTomato (right) to point out the neurite which will become the future axon. Live imaging was performed using a spinning disk confocal microscope to record movement of Stx6-GFP-labeled vesicles. Kymographs (lower panels) illustrate the transport of Stx6-GFP to the minor neurites. Anterograde transport events were traced as a line with a positive slope. There was extensive Stx6 transport to the minor neurite which will become the future axon. b As a control, stage 2 neurons were co-electroporated with the dendritic marker TfR-GFP (left) and KIF5 $\mathrm{C}^{560}$ tdTomato (right). Live imaging was performed as described in a. Kymographs (lower panels)

Meta-Morph software (Molecular Devices, LLC, Sunnyvale, CA). In some cases, the images were analyzed using ImageJ free software (http://imagej.nih.gov/ij/). All images were processed using Adobe PhotoShop (Adobe Systems, San Jose, CA, USA).

\section{KIF5C Rescue Experiments}

To perform these experiments, we titrated levels of the KIF5C plasmid to determine the lowest concentration necessary to restore KIF5C expression. Under these conditions, cells transfected with KIF5C shRNA, together with mKIF5CRFP plasmid, respectively, exhibited protein levels that were similar to those of control non-transfected neurons.

\section{Gel Electrophoresis and Western Blot}

Proteins were analyzed by SDS-PAGE. The concentration of acrylamide of the resolving gel varied from 7.5 to $15 \%$. The resolved proteins were transferred to nitrocellulose
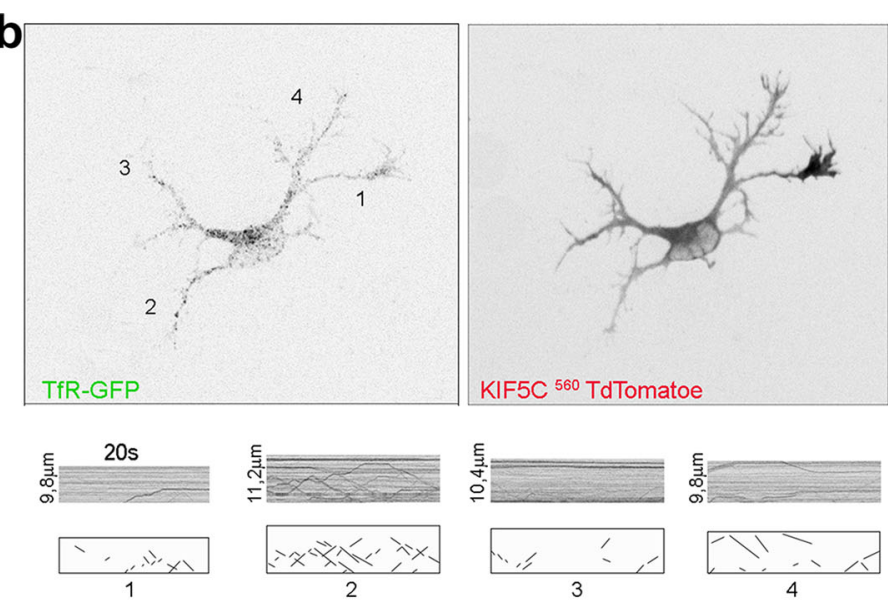

illustrate the transport of TfR-GFP to the minor neurites. Note that TfRGFP vesicles were transported preferentially to the neurites not enriched in KIF5C. c Quantification of anterograde transport events of Stx6-GFP vesicles (+s.e.m.-black bars) or TfR-GFP vesicles (empty bars) to different neurites. In the neurite with high accumulation of KIF5C ${ }^{560} \mathrm{TdT}$, the amount of Stx 6 transport increased significantly $(* p \leq 0.0001$ by one way ANOVA post-Hoc Tukey). In contrast, TfR-GFP is transported preferentially to the remaining neurites (not containing KIF5 $\mathrm{C}^{560} \mathrm{TdT}$ ). Contrast was inverted and non-linearly adjusted to improve visibility of dim moving vesicles. Neurons were imaged after $12 \mathrm{~h}$ DIV; $n=8$ cells for each condition. Calibration bar $=30 \mu \mathrm{m}$

membranes (AmershamHybond-ECL, GE Healthcare) in Tris-glycine buffer containing $20 \%$ methanol. The membranes were first dried, washed with Tris-buffered saline (TBS) $(10 \mathrm{mM}$ Tris, $\mathrm{pH} 7.5,150 \mathrm{mM} \mathrm{NaCl})$ and then blocked or directly blocked for $1 \mathrm{~h}$ in TBS containing $5 \%$ BSA. The blots were incubated with the primary antibodies in TBS containing $0.05 \%$ Tween 20 and $1 \%$ non-fat milk, for $12 \mathrm{~h}$ at $4{ }^{\circ} \mathrm{C}$. After washing with TBS containing $0.05 \%$ Tween 20 , the membranes were incubated with Odyssey IRdye CW 800 secondary antibodies (LI-COR Biosciences) for $1 \mathrm{~h}$ at room temperature. After washing, the blots were imaged using an Odyssey Infrared Imaging System (LI-COR Biosciences).

\section{Immunoprecipitation Assays}

Intact microsomal vesicles were incubated overnight at $4{ }^{\circ} \mathrm{C}$ with anti-KIF5C antibody. Immunoprecipitation was achieved by using protein A plus-coated beads (Protein A-Sepharose 4B Fast Flow-SIGMA). In order to discard non-specific binding, we made a control condition without antibody. 


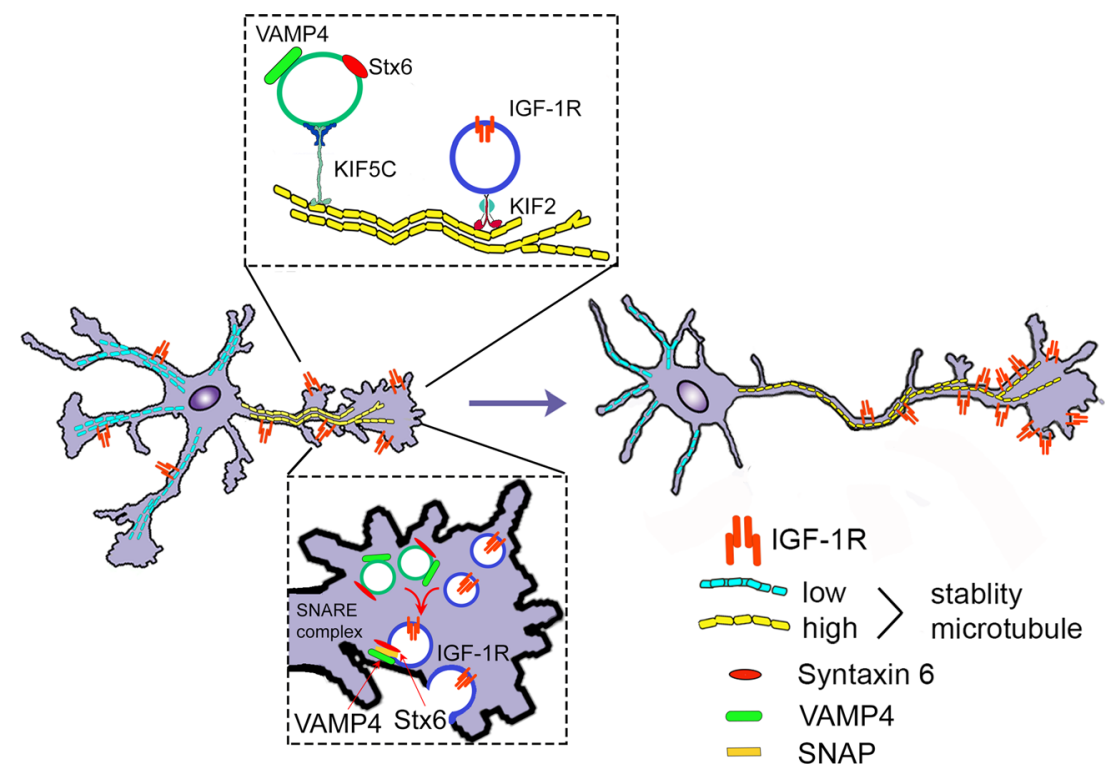

Fig. 8 KIF5C provides a mechanistic link between the requirement for stable microtubules, IGF-1r membrane insertion, and neuronal polarization. We hypothesize the following mechanism: moving on acetylated (stable) microtubules, the microtubular motor KIF5C transports the SNARE proteins Stx6 and VAMP4 preferentially to the neurite where stable microtubules are enriched. IGF-1r is transported by KIF2 to all the neurites ([13] see Fig. 1S). Stimulation with IGF-1 triggers the

After incubation, immunoprecipitation mixtures were spun to 4000 RPM during $10 \mathrm{~min}$, supernatant (SN) and immunoprecipitated (IP) were separated, and samples were processed for further analysis by SDS-PAGE/Western Blot.

\section{Immunofluorescence of Active IGF-1r}

Cells were cultured as previously described and transfected with KIF5C shRNA before plating (i.e., in suspension, at time 0 of culture). After $10 \mathrm{~h}$ in culture, cells were deprived of growth factors for $4 \mathrm{~h}$ and subsequently stimulated for $5 \mathrm{~min}$ with $20 \mathrm{nM}$ IGF-1, fixed and processed for immunofluorescence using an antibody selective for the phosphorylated form of IGF-1r [2] or an antibody that recognizes both the phosphorylated and non-phosphorylated forms of IGF-1r (anti- $\beta g c,[11]$ ).

\section{Imaging Vesicle Transport}

Coverslips with attached neurons (electroporated with Lonza kit P3 primary Cell 4D-Nucleofector X Kit L (Cat. No. V4XP-3024)) were loaded into a sealed heated chamber in phenol red free imaging medium (Hank's balanced salt solution buffered to $\mathrm{pH} 7.4$ with $10 \mathrm{mM}$ HEPES and supplemented with $0.6 \%$ glucose). Exposures were streamed continuously for $20 \mathrm{~s}$ or longer. The spinning disk microscope consisted of a Yokogawa CSU-10 spinning disk confocal head mounted on a Nikon TE2000 inverted microscope with a 60/1.45 NA formation of a population of vesicles containing Stx6, VAMP4, and IGF-1r [7]. Subsequently, these vesicles undergo exocytosis and thereby membrane insertion of IGF-1r. Once inserted into the plasmalemma, IGF1r can be activated by its cognate ligand, thereby stimulating more of its own exocytosis and regulating initial axonal outgrowth and establishment of neuronal polarity

Plan Apo objective and illumination from an Innova 70C Spectrum ion laser (Coherent). Streams of images (200300 ms exposures) were captured using an Orca ER CCD camera (Hamamatsu Instruments).

\section{Vesicle Transport Analysis}

Kymographs were generated using the Meta-Morph software. Contrast was inverted, so fluorescent organelles in the original image corresponded to dark lines in the kymograph. To measure transport, straight lines were traced on the kymographs to approximate each vesicle trajectory and the coordinates of the lines were transferred to an Excel spreadsheet to calculate the velocity and run length of each transport event. We also determined the total number of transport events.

\section{Subcellular Fractionation and Sucrose Density Gradient Centrifugation}

Fractions from E18 rat cerebral cortex were prepared as described previously [13]. In brief, rat cerebral cortex was gently homogenized with 10 volumes of ice-cold $0.32 \mathrm{M}$ sucrose, $10 \mathrm{mM}$ Hepes, $\mathrm{pH}$ 7.4. The homogenate was centrifuged at low speed $(3000 \times g)$ for $10 \mathrm{~min}$ at $4{ }^{\circ} \mathrm{C}$. The supernatant was centrifuged at medium speed $(9200 \times g)$ for $15 \mathrm{~min}$. The medium speed supernatant was again centrifuged at high speed $(100,000 \times g)$ for $60 \mathrm{~min}$ to yield a cytosolic fraction (S3) and a microsomal one (P3). The microsomal fraction was used for 
immunoprecipitation assays. For some experiments, the microsomal fraction was further applied to a continuous sucrose gradient $(0.3-1.6 \mathrm{M})$ at $48,000 \mathrm{rpm}$ for $2 \mathrm{~h}$ in a Sorvall STS 60.4 rotor. Fractions were then centrifuged at $100,000 \times g$, and the resulting pellets resuspended in Laemmli buffer. The same volume from each fraction was applied to SDS-PAGE and transferred to nitrocellulose membranes. Fractions were then analyzed by immunoblotting with antibodies to KIF5C, VAMP4, Stx6, and SNAP23.

\section{Animals}

Time-pregnant Wistar rats were used. All animal procedures were done using approved protocols by the Board of Animal Welfare, School of Chemical Sciences, National University of Córdoba.

Acknowledgments This work was supported by grants from the Agencia Nacional de Promoción Científica y Tecnológica, Argentina, PICT 1554 and 1646 (to SQ) and by the Secretaría de Ciencia y Técnica de la Universidad Nacional de Córdoba (SECYT-UNC-to SQ). We are indebted to Dr. Jennifer Petersen and Dr. Daniel Choquet for their help with the spinning disk microscopy experiments and to Dr. Corinne Lasmezas for critical reading of the manuscript.

\section{Compliance with Ethical Standards}

Competing Interests The authors declare that they have no competing interests.

\section{References}

1. de Anda FC, Meletis K, Ge X, Rei D, Tsai LH (2010) Centrosome motility is essential for initial axon formation in the neocortex. $\mathrm{J}$ Neurosci 30(31):10391-10406. doi:10.1523/JNEUROSCI.038110.2010

2. Sosa L, Dupraz S, Laurino L, Bollati F, Bisbal M, Caceres A, Pfenninger KH, Quiroga S (2006) IGF-1 receptor is essential for the establishment of hippocampal neuronal polarity. Nat Neurosci 9(8):993-995. doi:10.1038/nn1742

3. Caceres A, Ye B, Dotti CG (2012) Neuronal polarity: demarcation, growth and commitment. Curr Opin Cell Biol 24(4):547-553. doi:10.1016/j.ceb.2012.05.011

4. Shi SH, Jan LY, Jan YN (2003) Hippocampal neuronal polarity specified by spatially localized mPar3/mPar6 and PI 3-kinase activity. Cell 112(1):63-75

5. Nishimura T, Yamaguchi T, Kato K, Yoshizawa M, Nabeshima Y, Ohno S, Hoshino M, Kaibuchi K (2005) PAR-6-PAR-3 mediates Cdc42-induced Rac activation through the Rac GEFs STEF/Tiam1. Nat Cell Biol 7(3):270-277. doi:10.1038/ncb1227

6. Dupraz S, Grassi D, Bernis ME, Sosa L, Bisbal M, Gastaldi L, Jausoro I, Caceres A et al (2009) The TC10-Exo70 complex is essential for membrane expansion and axonal specification in developing neurons. J Neurosci 29(42):13292-13301. doi:10.1523 /jneurosci.3907-09.2009

7. Grassi D, Plonka FB, Oksdath M, Guil AN, Sosa LJ, Quiroga S (2015) Selected SNARE proteins are essential for the polarized membrane insertion of igf- 1 receptor and the regulation of initial axonal outgrowth in neurons. Cell Discovery 1:15023. doi:10.1038 /celldisc.2015.23 http://www.nature.com/articles/celldisc201523 \#supplementary-information

8. Witte H, Neukirchen D, Bradke F (2008) Microtubule stabilization specifies initial neuronal polarization. J Cell Biol 180(3):619-632. doi:10.1083/jcb.200707042

9. Jacobson C, Schnapp B, Banker GA (2006) A change in the selective translocation of the Kinesin-1 motor domain marks the initial specification of the axon. Neuron 49(6):797-804. doi:10.1016/j. neuron.2006.02.005

10. Nakata T, Hirokawa N (2003) Microtubules provide directional cues for polarized axonal transport through interaction with kinesin motor head. J Cell Biol 162(6):1045-1055. doi:10.1083 jjcb.200302175

11. Quiroga S, Garofalo RS, Pfenninger KH (1995) Insulin-like growth factor I receptors of fetal brain are enriched in nerve growth cones and contain a beta-subunit variant. Proc Natl Acad Sci U S A 92(10):4309-4312

12. Bernis ME, Oksdath M, Dupraz S, Nieto Guil A, Fernandez MM, Malchiodi EL, Rosso SB, Quiroga S (2013) Wingless-type family member $3 \mathrm{~A}$ triggers neuronal polarization via cross-activation of the insulin-like growth factor-1 receptor pathway. Front Cell Neurosci 7. doi:10.3389/fncel.2013.00194

13. Morfini G, Quiroga S, Rosa A, Kosik K, Caceres A (1997) Suppression of KIF2 in PC12 cells alters the distribution of a growth cone nonsynaptic membrane receptor and inhibits neurite extension. J Cell Biol 138(3):657-669

14. Xu XH, Deng CY, Liu Y, He M, Peng J, Wang T, Yuan L, Zheng ZS et al (2014) MARCKS regulates membrane targeting of Rab10 vesicles to promote axon development. Cell Res 24(5):576-594. doi:10.1038/cr.2014.33

15. Petersen JD, Kaech S, Banker G (2014) Selective microtubulebased transport of dendritic membrane proteins arises in concert with axon specification. J Neurosci 34(12):4135-4147. doi:10.1523/JNEUROSCI.3779-13.2014

16. Ozdinler PH, Macklis JD (2006) IGF-I specifically enhances axon outgrowth of corticospinal motor neurons. Nat Neurosci 9(11): 1371-1381. doi:10.1038/nn1789

17. Conde C, Caceres A (2009) Microtubule assembly, organization and dynamics in axons and dendrites. Nat Rev Neurosci 10(5): 319-332. doi:10.1038/nrn2631

18. Hirokawa N, Niwa S, Tanaka Y (2010) Molecular motors in neurons: transport mechanisms and roles in brain function, development, and disease. Neuron 68(4):610-638. doi:10.1016/j. neuron.2010.09.039

19. Stiess M, Bradke F (2011) Neuronal polarization: the cytoskeleton leads the way. Dev Neurobiol 71(6):430-444. doi:10.1002 /dneu.20849

20. Sakakibara A, Ando R, Sapir T, Tanaka T (2013) Microtubule dynamics in neuronal morphogenesis. Open Biol 3(7):130061. doi:10.1098/rsob.130061

21. Nakata T, Hirokawa N (2007) Neuronal polarity and the kinesin superfamily proteins. Sci STKE 2007(372):pe6. doi:10.1126 /stke.3722007pe6

22. Bradke F, Dotti CG (1999) The role of local actin instability in axon formation. Science 283(5409):1931-1934

23. Oliva AA Jr, Atkins CM, Copenagle L, Banker GA (2006) Activated c-Jun N-terminal kinase is required for axon formation. J Neurosci 26(37):9462-9470. doi:10.1523/JNEUROSCI.262506.2006

24. Benitez MJ, Sanchez-Ponce D, Garrido JJ, Wandosell F (2014) Hsp90 activity is necessary to acquire a proper neuronal polarization. Biochim Biophys Acta 1843(2):245-252. doi:10.1016/j. bbamcr.2013.11.013

25. Poirier K, Lebrun N, Broix L, Tian G, Saillour Y, Boscheron C, Parrini E, Valence S et al (2013) Mutations in TUBG1, DYNC1H1, 
KIF5C and KIF2A cause malformations of cortical development and microcephaly. Nat Genet 45(6):639-647. doi:10.1038/ng.2613

26. Sadler JB, Bryant NJ, Gould GW (2015) Characterization of VAMP isoforms in 3T3-L1 adipocytes: implications for GLUT4 trafficking. Mol Biol Cell 26(3):530-536. doi:10.1091/mbc.E14-09-1368

27. Wang S, Liu Y, Adamson CL, Valdez G, Guo W, Hsu SC (2004) The mammalian exocyst, a complex required for exocytosis, inhibits tubulin polymerization. J Biol Chem 279(34):35958-35966. doi:10.1074/jbc.M313778200

28. Brymora A, Valova VA, Larsen MR, Roufogalis BD, Robinson PJ (2001) The brain exocyst complex interacts with RalA in a GTPdependent manner: identification of a novel mammalian Sec3 gene and a second Sec15 gene. J Biol Chem 276(32):29792-29797. doi:10.1074/jbc.C100320200

29. Walter AM, Kurps J, de Wit H, Schoning S, Toft-Bertelsen TL, Lauks J, Ziomkiewicz I, Weiss AN et al (2014) The SNARE protein vtila functions in dense-core vesicle biogenesis. EMBO J 33(15): 1681-1697. doi:10.15252/embj.201387549

30. Golebiewska EM, Harper MT, Williams CM, Savage JS, Goggs R, Fischer von Mollard G, Poole AW (2015) Syntaxin 8 regulates platelet dense granule secretion, aggregation, and thrombus stability. J Biol Chem 290(3):1536-1545. doi:10.1074/jbc.M114.602615

31. Tanabe K, Tachibana T, Yamashita T, Che YH, Yoneda Y, Ochi T, Tohyama M, Yoshikawa H et al (2000) The small GTP-binding protein TC10 promotes nerve elongation in neuronal cells, and its expression is induced during nerve regeneration in rats. $\mathrm{J}$ Neurosci 20(11):4138-4144

32. Khan IA, Luduena RF (1996) Phosphorylation of beta III-tubulin. Biochemistry 35(12):3704-3711. doi:10.1021/bi951247p

33. An S, Tsai C, Ronecker J, Bayly A, Herzog ED (2012) Spatiotemporal distribution of vasoactive intestinal polypeptide receptor 2 in mouse suprachiasmatic nucleus. J Comp Neurol 520(12):2730-2741. doi:10.1002/cne. 23078

34. Kreis TE (1987) Microtubules containing detyrosinated tubulin are less dynamic. EMBO J 6(9):2597-2606

35. Beirowski B, Gustin J, Armour SM, Yamamoto H, Viader A, North BJ, Michan S, Baloh RH et al (2011) Sir-two-homolog 2 (Sirt2) modulates peripheral myelination through polarity protein Par-3/ atypical protein kinase C (aPKC) signaling. Proc Natl Acad Sci U S A 108(43):E952-E961. doi:10.1073/pnas.1104969108 